\title{
Theory of Analogous Force on Number Sets
}

\author{
Enrique Canessa* \\ The Abdus Salam International Centre for Theoretical Physics, Trieste, Italy
}

\begin{abstract}
A general statistical thermodynamic theory that considers given sequences of $x$-integers to play the role of particles of known type in an isolated elastic system is proposed. By also considering some explicit discrete probability distributions $p_{x}$ for natural numbers, we claim that they lead to a better understanding of probabilistic laws associated with number theory. Sequences of numbers are treated as the size measure of finite sets. By considering $p_{x}$ to describe complex phenomena, the theory leads to derive a distinct analogous force $f_{x}$ on number sets proportional to $\left(\frac{\partial p_{x}}{\partial x}\right)_{T}$ at an analogous system temperature $T$. In particular, this yields to an understanding of the uneven distribution of integers of random sets in terms of analogous scale invariance and a screened inverse square force acting on the significant digits. The theory also allows to establish recursion relations to predict sequences of Fibonacci numbers and to give an answer to the interesting theoretical question of the appearance of the Benford's law in Fibonacci numbers. A possible relevance to prime numbers is also analyzed.
\end{abstract}

PACS numbers: 02.50.-r, 05.70.Ce, 89.90.+n

\footnotetext{
*E-mail: canessae@ictp.trieste.it
} 


\section{INTRODUCTION}

There are suggestive parallels between various aspects of number theory and physics phenomena. The connection often involves affinity with functions arising in statistical mechanics 1]. For example, a connection between given number sets and the thermodynamic formalism has been established for the multifractal spectrum via a Legendre transform [2] and the statistical mechanics applications for prime numbers via the Riemann partition function [3]. Bose condensation has been indirectly linked to the zeta function in [4]. Primes as such have also attracted recent applications in acoustics and dynamical systems [5]. Furthermore, the existence of an imaginary quantum mechanical potential for the prime numbers has been idealized in [6] after some energy considerations. In a similar context, additive number theory in terms of scale invariance has been used to study the origin and form of the uneven distribution of integers of many random data sets [7, 8, 9, 10, 11, 12].

In this paper we introduce an alternative approach which considers sets of $x$-natural numbers to play the role of particles of known type in an isolated elastic system and assume that their discrete probability distribution can be split as the product of some functions. By applying thermodynamics techniques, we develop an analogous statistical mechanics version of the average energy and energy states of arbitrary number sets.

From the Helmoltz free energy in a reversible isothermal process, a relation for an analogous tensile force $F>0$ is derived by treating $x$ as variations in length in the direction of $F$ and considering the number system as a elastic specimen (body). In particular, our theory allows to give an alternative physics answer to the uneven distribution of integers in terms of a screened inverse square force which ideally may act on these numbers. It also allows to establish second order polynomial recursions to predict sequences of integers following generalized Fibonacci numbers. We also analyze a possible application of the distinct forces to primes.

Hereafter, the word $x$-number is used to refer to quantities which are integers and positive. 


\section{UNDERLYING THEORY}

\section{A. A priori probability postulate}

Let us define the discrete probability distribution of finding a certain positive natural number $x$ as

$$
p_{x}=g_{1}(x) g_{2}(x)
$$

with $g_{1}(x)>0$ and $0<g_{2}(x) \leq 1$ and satisfying the normalization $\sum_{x} p_{x}=1$. Such discrete probability distribution functions (and also discrete mixtures of them), used to describe complex phenomena, are given by the product of two different functions. The real function $g_{2}(x)$ here represents the set of digital frequencies which remain fixed under base changes given by the real function $g_{1}(x)$.

This factorization may be seen somewhat arbitrary, however it is based on composed functions which include the Binomial(A,B), Furry or Geometrical, inversed Logarithmic, Pascal or Polya and Poisson distributions. Furthermore, a probability decomposition into (two) functions is no new. This type of factorization appears formally, e.g., in the analysis of stochastic processes on graphs. According to the well-known Hammersley-Clifford Theorem, a probability function $p(x)$ is formed by a normalized product of positive functions on cliques (i.e., fully connected subgraph) of the graph $\mathcal{G}$ when the stochastic process $x$ is Markov relative to $\mathcal{G}$.

To simplify notation in all later sums and products the variable $x$ represents an upper bound (or cutoff).

\section{B. Thermodynamic Analogy}

Using the above probability postulate the information theoretical entropy $S_{I}=$ $-\sum_{x} p_{x} \ln p_{x}$ then becomes

$$
S_{I}=-\sum_{x} g_{1}(x) g_{2}(x)\left\{\ln g_{1}(x)+\ln g_{2}(x)\right\}
$$

In order to make a thermodynamic analogy, let us consider next the Helmholtz free energy $A=E-T S$ assuming an analogous temperature $T$ and volume $V$ to be independent vari-

ables, with $E$ the analogous internal energy of number sets. Because of the close relationship 
of Eq.(2) to the thermodynamic entropy $S$, we can readily identify

$$
\begin{aligned}
& A / k T \rightarrow \sum_{x} p_{x} \ln g_{1}(x), \\
& E / k T \rightarrow-\sum_{x} p_{x} \ln g_{2}(x) .
\end{aligned}
$$

with $k$ the Boltzmann constant. Reduced units are used throughout. The choice of the latter quantities may be seen quite arbitrary, but as mentioned in the conclusion these can be justified in analogy with standard thermodynamics results.

In this case, for the correct application of conventional thermodynamics the normalization of $p_{x}$ is used to define both the Boltzmann and the Shannon entropies.

For 'non-interacting' number sets $x>1$, we can get an approximated statistical mechanics version of the average energy as the sum of individual contributions $E \rightarrow \bar{E}=\sum_{x} \bar{n}_{x} \epsilon_{x}$. The total number of positive natural number $x$ 's is given by $N=\sum_{x} \bar{n}_{x}$ and $\epsilon_{x}$ represents their analogous energy states. Thus, from Eqs.(1) and (4) we further identify

$$
\begin{aligned}
\bar{n}_{x} & \rightarrow-\ln g_{2}(x) \geq 0 \\
\epsilon_{x} / k T & \rightarrow g_{1}(x) g_{2}(x)=p_{x} \geq 0 .
\end{aligned}
$$

The number of states available to a molecular system is given by the partition function $\Omega$ which satisfies the relation $S=k \ln \Omega(N, V, E)$ in the microcanonical ensemble and relates the canonical partition function by $Q(N, V, T)=\Omega(N, V, E) e^{-E / k T}$. Therefore, from Eqs.(2), (41) and (6) we also have in analogy

$$
Q_{x}=\prod_{x}\left[g_{1}(x)\right]^{-p_{x}}=\prod_{x}\left[g_{1}(x)\right]^{-\epsilon_{x} / k T} .
$$

As expected, the form of our canonical partition function depends on the number probability distribution of Eq.(11).

\section{Analogous Force}

To derive a relation for an analogous external tension which we may ideally apply on number sets in a gedanken experiment, let us treat $x$ as a variation in length in the direction of a tensile force $f_{x}$ and the number system as an elastic body. As a first approximation, the required relation is then obtainable directly from the first and second laws of thermo-

dynamics in a reversible isothermal process [13]. For a change at constant $T$, it follows that 
$d A=d W=f d \ell$, where $W$ is the work done on the system by its surroundings in a small elongation $d \ell$. Hence, by partial differentiation of Eq.(3),

$$
f_{x}=\left(\frac{\partial A}{\partial x}\right)_{T}=\frac{\epsilon_{x}}{g_{1}(x)}\left(\frac{\partial g_{1}(x)}{\partial x}\right)_{T}+\ln g_{1}(x)\left(\frac{\partial \epsilon_{x}}{\partial x}\right)_{T} .
$$

Since the free energy depends on the upper cutoff $x$ then the derivative was taken in terms of that variable, ii.e. the derivative of the last term in the sums. If $f_{x}<0$, this corresponds to an analogous compressive force on the number system. We shall show next that this new tensile force may indeed be useful to characterize number sets.

\section{PROBABILITY DISTRIBUTIONS AND RELATED FUNCTIONS}

\section{A. First Digits Patterns: $g_{1}(x) \equiv c_{0}, g_{2}(x) \equiv f[\ln x]$}

In terms of the first digits of unbiased random samples from random distributions, the so-called Benford's law follows the logarithmic distribution

$$
p_{x}=\log _{10}\left(1+\frac{1}{x}\right) \equiv c_{0} \ln \gamma(x)
$$

with $1 / c_{0} \equiv \ln 10, \gamma(x)=1+\frac{1}{x}$ and $x=1,2, \cdots$. This empirical law deals with the distribution of first digits in numbers in which significant positive digits are not uniformly distributed, but smaller digits and smaller combinations of significant digits are favored.

The sum of probabilities in this case is

$$
\sum_{x} p_{x} \equiv \beta_{x}=c_{0} \sum_{x} \ln \gamma(x)=c_{0} \ln \prod_{x}\left(\frac{x+1}{x}\right)=c_{0} \ln (1+x) .
$$

This result implies that $p_{x}$ is not a proper probability since it imposes some maximum value 7].

From Eq. (17) the evaluation of the analogous partition function is straight forward, namely

$$
Q_{x}=\prod_{x} \gamma(x)^{-c_{0} \ln c_{0}}=(1+x)^{-c_{0} \ln c_{0}} .
$$

According to Eq.(8), our analytical expression for the analogous force acting on this number set becomes

$$
\frac{f_{x}}{c_{0} \ln c_{0}}=-\frac{1}{x+x^{2}} .
$$

It is from this base-invariant behavior of a distinct $f_{x}$ that we can make an attempt to understand the significant digit distribution of Eq.(9) in terms of an screened inverse square force. 


\section{B. Relevance to Primes: $g_{1}(x) \equiv e^{-1}, g_{2}(x) \equiv x^{-\sigma}$}

If we generalize the above to a power law behavior for the sequence of primes only greater than one with an exponent $\sigma>1$, i.e., $p_{x_{p}}=1 /\left(e x_{p}^{\sigma}\right)$, then the class of analogous forces on prime numbers becomes

$$
f_{x_{p}}=\frac{\sigma}{x_{p}^{\sigma+1}}
$$

-a fingerprint for primes.

Using Eq.(17), the analogous partition function approaches

$$
\frac{Q_{x_{p}}}{e^{1 / e}} \approx \prod_{x_{p}>1} \frac{1}{1-x_{p}^{-\sigma}}=\sum_{x} \frac{1}{x^{\sigma}} .
$$

Another possible relation of our statistical thermodynamic approach to prime numbers follows by considering the average system energy. In the limit of Eq.(12), this takes the form

$$
\frac{E}{c_{0}} \approx \sum_{x} \frac{\ln x}{x}
$$

In principle this could be asymptotic to $\sum_{x} \frac{1}{\pi(x)}$, where the counting function $\pi(x)$ denotes the number of prime numbers $x_{p}$ that do not exceed $x[14]$.

\section{DISCUSSION}

\section{A. Benford's law, Analogous Force and Thermodynamics}

As an example, in Fig 1 we plot the probability distribution and our distinct force on (up to 300) numbers from Fibonacci series and compare results with the present analogous statistical thermodynamics approach. The theoretical calculation of the analogous force is obtained using Eq.(12) (and its large- $x$ behavior). The numerical calculation of $f_{x}$ for Fibonacci numbers is also rather simple. This is derived using Eq.(8) and from the fact that $c_{x}$ is constant. By the two-point central formula we approximate

$$
f_{x} / \ln c_{0}=\left(\frac{\partial p_{x}}{\partial x}\right)_{T} \approx \frac{1}{2}\left(p_{x+1}-p_{x-1}\right) .
$$

This means that the probability distribution $p_{x}$ is related to an analogous local potential field at each number (i.e., particle) as in the well known diffusion limited aggregation model 15]. Assuming a sort of superposition principle, the total analogous force $F \equiv \sum_{x} f_{x}$ can also be readily evaluated. From Eq.(12) we obtain $F / c_{0}=-\ln c_{0} / \gamma(x)$. 
Since $c_{0} \approx 0.4343$ in logarithm base 10 , we then have an analogous system entropy $S>0$, the analogous Helmholtz free energy $A<0$, the analogous partition function $Q_{x}>0$, an analogous expansive force on the integers $f_{x}>0$ and the sum of probabilities $\beta_{x}>0$. Interestingly, from Eq.(10) we also have that this sum only becomes normalized at $x=9$ (i.e., a decadelong). On the other hand, the increase in the analogous entropy as $x$ increases follows the natural tendency of thermodynamic systems to move toward disordered states having more microscopic states characterized by our analogous macroscopic variables.

Besides, the particular $\gamma(x)$ under consideration allows to derive a measure for an analogous inverse temperature of the integers system. From Eqs.(21), (41) and (9), we thus approximate

$$
\frac{\Delta(S / k)}{\Delta(E / k T)}=1+\frac{\ln c_{0}}{\ln (\ln \gamma(x))}
$$

where $\Delta$ represents functions difference between consecutive number fluctuations.

For $x>>1$, Eq.(12) implies that $p_{x} \approx c_{0} x^{-1}$. Such a power law behavior with exponent one is the solution of the functional form $p_{x^{\prime}}=p_{a x}=A_{a} p_{x}$ which may correspond to an analogous scale invariance [10].

The results shown in the figure allows to argue that our simple physics-based approach is not merely speculative. Indeed it yields to a different and more complete understanding of Benford's law than that of previous attempts [9, 10, 11, 12]. It includes scale invariance, frequencies behavior under base changes and explanation for a logarithmic law distribution besides the analogous thermodynamic characterization of given number sets such as time series or primes.

\section{B. Screening}

For $x>>1$, the force $\frac{f_{x}}{c_{0} \ln c_{0}}$ can be approximated as $-\frac{e^{-\frac{1}{x}}}{x^{2}}$, so we could consider the exponential term as being screening. The presence of $\exp (-1 / x)$ screening in this analogous inverse-squared force implies that the second leading digits (i.e., $10 \leq x \leq 99$ ) are much more uniformly distributed than the first digits, and the third than the second, and so on according to observations [7]. This is reflected by the numerical differences $f_{1}-f_{9}=0.17708$, $f_{10}-f_{99}=0.00326, f_{100}-f_{999}=0.00004$. The main message is that the asymmetry in favor of smaller significant digits or combinations of significant digits can be understood in terms of analogous screened inverse square force acting on the number sets. 


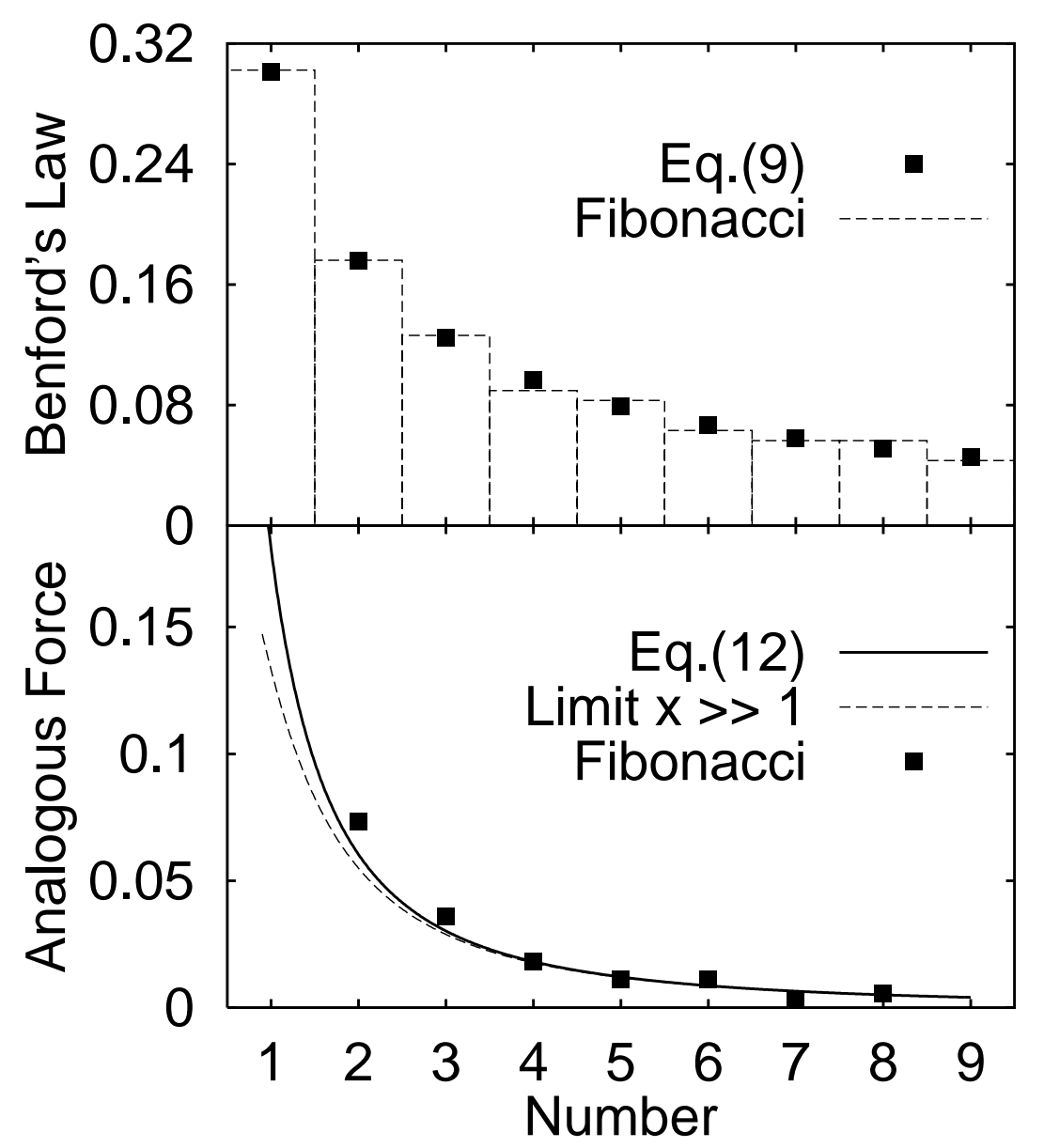

FIG. 1: Probability distribution of, and distinct force on, Fibonacci numbers.

Indeed screening could be a way to unveil the fact that significant digits are not independent, but that instead knowledge of one digit affects the likelihood of another. Furthermore, since screening is a consequence of having the base invariant probability distribution of Eq.(11), then the choice of digital frequencies bounded by $1<1+\frac{1}{x} \leq e$ leads to support a logarithmic law distribution. 


\section{Recursions: A Possible Scenario for Number Prediction}

From a physical point of view, the equilibrium of forces within defect-free plate under external shear can be approximated by the discrete Laplace equation [16, 17, 18]

$$
\sum_{k=1}^{4} G_{k} \Delta u_{k}=0
$$

where $\Delta u_{k}=u_{k}(i)-u(i)$ denotes relative displacements of the $i$ th lattice point with respect to its nearest neighbors and $G_{k}$ is the corresponding modulus of rigidity of the bond connecting the $i$ th lattice point to its neighbor.

From Eq.(18) it follows then that for a 1D system

$$
u(i)=\hat{G}_{1} u_{1}(i)+\hat{G}_{2} u_{2}(i),
$$

where $\hat{G}_{i}=G_{i} / \sum_{i} G_{i}(i=1,2)$.

It is not unreasonable to assume that the moduli of rigidity of our analogous elastic system are proportional to each other, at least prior to any equivalent breakdown condition which may prevail. Therefore if we consider $u_{1}(i) \propto\left(G_{2} / G_{1}\right) u_{2}(i-1)$, then we have that all displacements $u_{2}, u_{1}$ and $u$ follow generalized fibonacci sequences of the form [19]

$$
\mathcal{U}(i+2)=\mathcal{U}(i)+\mathcal{U}(i+1)
$$

Within our thermodynamics framework, the sequences of $x$-integers played the role of analogous 'non-interacting' particles. Besides, their magnitude may be seen as representing the above effective (increasing) displacements, say expected values $x=\mathcal{E}[\mathcal{U}]$, at different times $i$. The analogous force may be in turn seen as an applied shear acting on our 1D isolated elastic system of finite size $x$, which is being pulled out (or in).

Therefore following these ideas from fracture phenomena, a possible scenario to deduce second order polynomial recursions leading to predict sequences of numbers becomes possible.

\section{CONCLUDING REMARKS}

In this paper we have introduced a quite abstract statistical mechanical system whose states are represented by natural numbers. By considering some explicit probability distributions for the natural numbers, we claim that they lead to a better understanding of probabilistic laws associated with number theory. 
Within this kind of thermodynamic formalism we have investigated probability distributions which lead to Benford's law. Sequences of numbers have been treated as the size measure of finite sets and, in analogy with statistical thermodynamics, they have been assumed to play the role of particles of known type in an isolated elastic system.

The relation to physics is rather in the thermodynamical formalism and terminology for number theoretical problems. The assignment of statistical quantities here proposed are not arbitrary. Our analogies for the considered functions, e.g., Helmoltz free energy, etc, follows the similar thermodynamical approach as proposed within Multifractal physics. The expression of the Shannon entropy in Eq.(2) split into two terms with the identification of one of the terms with an analogous free energy and the other with an analogous internal energy can be justified (although not rigorously) in terms of the form of our canonical partition function which depends on the number probability distribution of Eq. (11) as may be expected. Furthermore as derived from such identification, we can argue that the relation in Eq.(17) tends to unity in the large $x$-limit in analogy with the standard thermodynamic definition $T=\left(\frac{\partial E}{\partial S}\right)_{V, N}$.

Though this scheme may be seen as rather abstract, we have shown that it leads to very interesting connections with statistical thermodynamics and allows to interpret physically the observed probability distribution of first digits in terms of a stretching force (tension) which ideally may pull integers number sets along analogous lengths.

The introduction of an analogous force within our formalism has been done on a formal level. The new insights gained by such a formal introduction and its utility include, in particular, the study of the dynamics of given number sets by establishing second order polynomial recursions to predict sequences of generalized Fibonacci numbers. This gives an answer to the interesting theoretical question of the appearance of the Benford's law in Fibonacci numbers.

Following recent interest in Benford's law [7], we believe the new force may find application to detect fraud in financial data [20], by looking for anomalies in their analogous macroscopic thermodynamic variables, in the use of logarithmic computers to speed calculations [21], by analyzing the screening behavior of the analogous inverse square forces on floating points, or in the characterization of the hopcount in Internet [22]. 


\section{Acknowledgement}

The author would like to thank the ARPL Group of the Abdus Salam ICTP, Trieste, for hospitality and support.

[1] G.E. Andrews et al. in "The Unreasonable effectiveness of Number Theory", Proceedings of Symposia in Applied Maths, Vol.46, ed. S.A. Burr (Amer. Math. Soc., 1992),

[2] J. Lee and H.E. Stanley, Phys. Rev. Lett. 26, 2945 (1988).

[3] M. Wolf, Phys. A, 274, 149 (1999).

[4] B. Julia, in "Number Theory and Physics", Springer Proceedings in Physics, Vol 47, Ed. J.M. Luck et al. (Springer-Verlag, Berlin, 1990, p.276).

[5] S.R. Dahmen, S.D. Prado and T. Stuermer-Daitx, Phys. A 296, 523 (2001).

[6] G. Mussardo, The Abdus Salam ICTP Preprint No 97/199 (1997); New Scientist, Jan 1998, p.18.

[7] T.P. Hill, Am. Sci. 86, 358 (1998); Stat. Sci. 10, 354 (1996).

[8] C.R. Tolle, J.L. Budzien and R.A. LaViolette, Chaos 10, 331 (2000).

[9] A.K. Adhikari and B.P. Sarkar, Sankhyã B 30, 47 (1968).

[10] see, e.g., http://mathworld.wolfram.com

[11] G.A. Gottwald and M. Nicol, Phys. A 303, 387 (2002).

[12] M.A. Snyder et al., Phys. Rev. E 64, 026222 (2002).

[13] L.R.G. Treloar, "The Physics of Rubber Elasticity" (Clarendon Press, Oxford, 1975).

[14] G. Tenenbaum and M. Mendès France, "The Prime Numbers and Their Distribution", (AMS, Presses Universitaires de France, Paris, 2000).

[15] T.A. Witten and L.M. Sander, Phys. Rev. Lett. 47, 1400 (1981).

[16] H. Takayasu, Prog. Theor. Phys. 74, 134 (1985).

[17] E. Canessa and B. Tanatar, Phys. Rev. A 44, 3471 (1991).

[18] E. Canessa and B. Tanatar, J. Mat. Sci. Lett.Phys. 11, 401 (1992).

[19] S.W. Golomb and A. Lempel, SIAM J. Appl. Math 33, 587 (1977).

[20] M. Nigrini, J. Am. Tax. Assoc. 18, 72 (1996).

[21] D.E. Knuth, "The Art of Computer Programming", Vol 2, (Reading, Mass., Addison-Wesley, 
1981, p.238).

[22] P. Van Mieghem, G. Hooghiemstra and R.W. van der Hofstad, Delft Univ. Tech. Report 2000125 (2000). 